УДК 622.24 .051 .64

\title{
ВЛИЯНИЕ СРЕДЫ ПРИЗАБОЙНОЙ ЗОНЫ СКВАЖИНЫ НА ЭФФЕКТИВНОСТЬ РАЗРУШЕНИЯ ГОРНОЙ ПОРОДЫ РЕЗЦОМ РDС
}

\author{
Нескоромных Вячеслав Васильевич', \\ sovair@bk.ru
}

\section{Попова Марина Сергеевна ${ }^{1}$,} alleniram83@mail.ru

\author{
Лиу Баочанг², \\ liubc@jlu.edu.cn \\ 1 Сибирский федеральный университет, \\ Россия, 660025, г. Красноярск, пр. им. газеты «Красноярский рабочий», 95. \\ 2 Университет Цзилинь, \\ Китай, 130061, г. Чанчунь, ул. Химинжу, 938.
}

\begin{abstract}
Актуальность. Породоразрушающий инструмент, армированный резцами $P D C$, зарекомендовал себя как буровой инструмент с хорошими эксплуатационными показателями. Как известно из практики бурения, инструмент такого типа при правильной технологии его применения способен обеспечить высокие скорости проходки скважин и ресурс. Поэтому одним из приоритетных направлений в развитии технических средств бурения является совершенствование конструкций инструмента типа PDC. Методы проектирования бурового инструмента нового поколения должны основываться на комплексном подходе к исследованию его работы на забое. Одним из основных фракторов, влияющих на эфффективность реализации породоразрушающего инструмента, является сопротивление забоя. Исследованию изменения физико-механических свойств геологического разреза с глубиной повящено большое количество трудов. А жидкая среда, образовавшаяся на забое скважины в результате процесса бурения, изучена слабо. Часто, при проведении научных исследований, наличием шлама в призабойной зоне пренебрегают. Однако, учитьвая особенности формы и размеры режущей части резцов PDC, сместь шлама и непрерывно движущейся промывочной жидкости может обладать определенной плотностью и создавать выталкивающее усилие, действующее на плоскость резца, и как следствие оказывать влияние на эффрективность работы бурового инструмента в целом. Поэтому актуальным вопросом является исследование свойств среды, состоящей из промывочного агента, шлама и разрушенной горной породы, и определение степени ее влияния на результаты работы бурового инструмента типа PDC.

Цель: определить степень влияния сил сопротивления среды, образовавшейся в призабойной зоне скважины, на процесс бурения скважин.

Методы: аналитический метод, метод компьютерного имитационного моделирования.

Результаты. Среда, образовавшаяся в призабойной зоне в процессе бурения скважины и состоящая из породы, шлама и промывочной жидкости, находящихся в различном состоянии, оказывает выталкивающее действие на резеи, что снижает величину углубления. Показатель степени сопротивления описанной среды определяется скоростью резания, уелом установки резца и плотностью ее составляющих. Полученные результаты могут быть использованы при конструировании бурового инструмента типа PDC. Изменение угла установки или формы резца может способствовать не только снижению сил сопротивления, но и улучшению разрушающей способности инструмента за счет разуплотнения породы путем интенсификации проникновения жидкости в породу.
\end{abstract}

\section{Ключевые слова:}

Скорость резания-скалывания породы, углубление, бурение, резец PDC,

сопротивление среды, глубина резания-скалывания, ядро сжатия, разрушение горной породы.

\section{Введение}

Породоразрушающий инструмент типа $P D C$ в последнее время имеет широкое применение в отрасли бурения различного назначения [1-25]. Это связано с хорошими эксплуатационными показателями данного инструмента [26, 27]. Поэтому актуальность совершенствования бурового инструмента, армированного поликристаллическими алмазными резцами, не исчерпана и нуждается в детальном исследовании механизма его работы на забое, который зависит от множества факторов.

Собственно, основной конструктивной особенностью бурового породоразрушающего инструмента типа $P D C$ и является сам резец, который отличается своими прочностными данными, имеет цилиндрическую форму и изготавливается различных размеров.
Устанавливаются такие резцы в буровом инструменте по определенной схеме. Каждый резец бурового инструмента имеет свой передний $\left(\gamma_{\text {п }}\right)$ и фронтальный $\left(\varphi_{\mathrm{p}}\right)$ угол установки. Как показывают аналитические исследования [5], значения данных углов оказывают существенное влияние на работоспособность инструмента в целом.

Эффективность работы бурового инструмента может определяться величиной углубления за оборот. Значение данного параметра бурения зависит от величины глубины резания-скалывания породы каждым отдельным резцом. При внедрении резца в горную породу основным препятствием по увеличению значения глубины резания-скалывания являются силы сопротивления, возникающие со стороны забоя. Причем, как показывают аналитические исследования 
[10], сопротивление резцу оказывает не только сама порода, но и среда, образовавшаяся в процессе бурения в призабойной зоне скважины. Значение глубины резания-скалывания породы резцом типа $P D C$ можно определить по формуле:

$$
h=\sqrt[2]{\left\{\frac{\left[\left(P-A \sin \gamma_{\mathrm{\pi}}\right)\left(1-f \operatorname{tg} \gamma_{\mathrm{\pi}}\right)-A \operatorname{tg} \gamma_{\mathrm{\pi}}\right] \sin \gamma_{\mathrm{c \kappa}}}{\pi \sigma_{\mathrm{c \kappa}} \sqrt{d} \operatorname{tg} \gamma_{\mathrm{\pi}} \cos \varphi_{\mathrm{p}}(1+\operatorname{tg} \varphi)}\right.},
$$

где $P$ - осевая нагрузка на алмаз, $\mathrm{H} ; \gamma_{\text {п }}$ - передний угол установки резца, град; $\gamma_{\text {ск }}$ - угол скалывания, град; $\sigma_{\text {ск }}$ предел прочности породы на скалывание, Па; $d$ - диаметр резца, м; $\varphi_{\mathrm{p}}-$ фронтальный угол установки резца, град; $\varphi$ - угол внутреннего трения породы, град; $f$ - коэффициент внешнего трения на контакте поверхность резца - горная порода; $A=0,125 v_{\mathrm{p}}^{2} \rho_{c} \pi d^{2} C_{c} \cos \gamma_{\Pi}$, где $v_{\mathrm{p}}-$ скорость резания-скалывания, м/с; $\rho_{c}-$ весовая плотность среды с учетом плотности бурового раствора, насыщенного шламом из зоны разрушения и разрушаемой горной породы, кг $/ \mathrm{M}^{3} ; C_{\mathrm{c}}-$ коэффициент сопротивления со стороны резца, определяемый формой плоскости резца

Согласно формуле (1), глубина резания-скалывания породы одним резцом $P D C$ помимо углов его установки определяется и другими факторами. Например, режимом бурения, который выражается значением осевой нагрузки на резец $(P)$ и скорости перемещения резца по забою $\left(v_{\mathrm{p}}^{2}\right)$; свойствами разрушаемой породы (коэффициентом внутреннего трения горной породы ( $\varphi)$ и прочностью горной породы на скалывание $\left.\left(\sigma_{\mathrm{ck}}\right)\right)$. На величину глубины резания-скалывания также оказывают влияние такие показатели, как диаметр резца $(d)$, угол скалывания породы $\left(\gamma_{\text {ск }}\right)$, коэффициент внешнего трения резца о породу $(f)$ и плотность жидкой среды, возникающей на забое скважины $\left(\rho_{c}\right)$.

Таким образом, регулируя перечисленные параметры, можно добиться наиболее эффективных ре- зультатов бурения породоразрушающим инструментом данного типа. Если геометрию инструмента (углы установки, диаметр и размеры резца) нельзя изменить в процессе бурения, то геолого-технологические условия бурения могут иметь периодический характер. Своевременное изменение режимов бурения возможно путем применения средств автоматизации контроля бурения. Единственным, не поддающимся внешнему регулированию, фактором, влияющим на результативность буровых работ, является физикомеханическая характеристика разрушаемых горных пород. Изучению свойств горных пород и их влиянию на процесс бурения посвящено много научных трудов, при этом образовавшейся на забое жидкой среде, состоящей из смеси промывочного агента и породы, уделяется незначительное внимание и только с целью улучшения очистки скважины. Полученные результаты аналитических исследований [10], указывающие на наличие выталкивающей функции среды, образованной в призабойной зоне скважины при бурении, требуют дополнительных исследований данного явления с учетом механизма разрушения горной породы резцом.

\section{Методика}

Согласно схеме механизма разрушения породы резцом типа $P D C$, представленной на рис. 1, на плоскость резца воздействует сопротивление жидкой среды, сформировавшейся над забоем $R_{\mathrm{p}}$, и сопротивление со стороны массива горной породы $R_{\text {п. Состояние }}$ указанных сред, оказывающих сопротивление, зависит от множества факторов. К примеру, степень разрушенности породы, ее насыщенность промывочной жидкостью, собственно сам вид применяемой в данный момент промывочной жидкости, степень анизотропности горной породы, скорость циркуляции жидкости на забое и т. д.

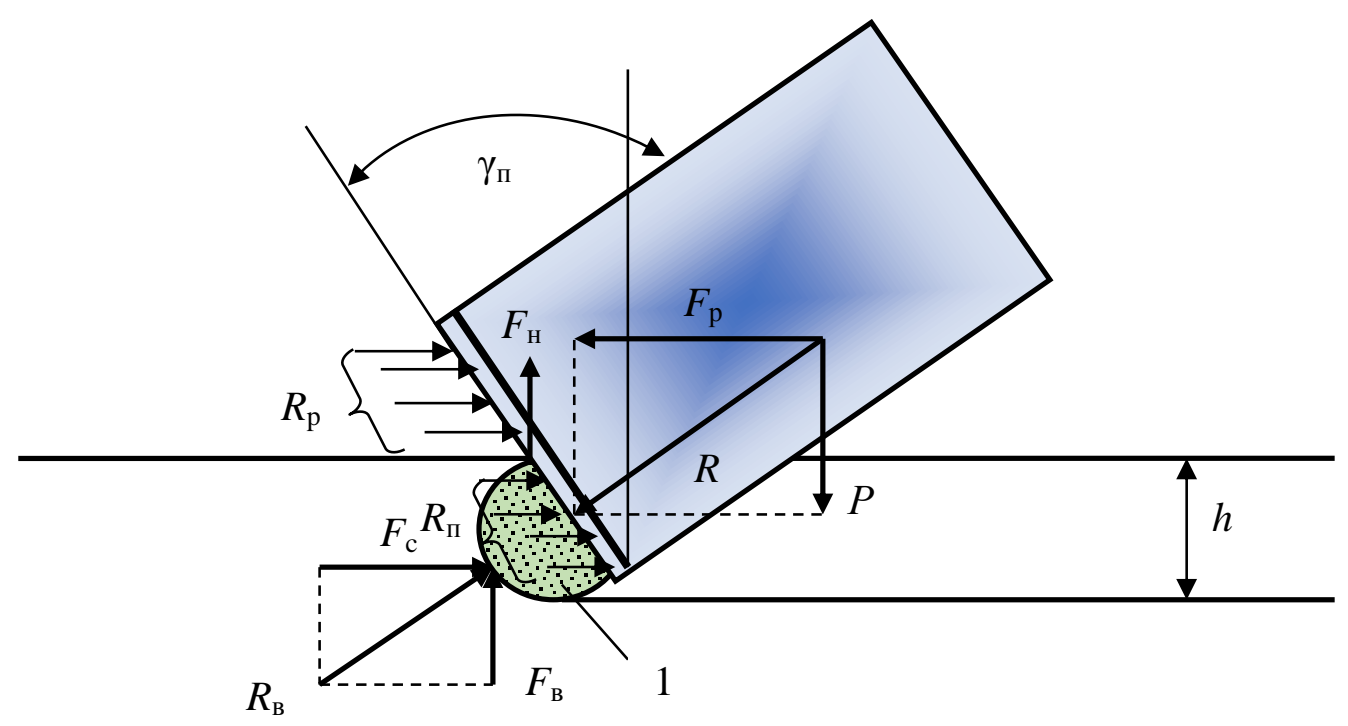

Pис. 1. Схема механизма разрушения горной породы резцом РDC: 1 -ядро сжатия породы

Fig. 1. Diagram of the mechanism of rock destruction with a PDC cutter: 1 -rock compression core 
При детальном рассмотрении области контакта резца $P D C$ с горной породой (рис. 2) можно выделить несколько основных зон, отличающиеся физикомеханическими свойствами, состоянием и составом. Согласно исследованию [5], при внедрении резца в породу перед его режущей поверхностью образуется ядро сжатия горной породы - 1. Разрушение сопровождается образованием линии скалывания, которая ориентируется от ядра в направлении поверхности забоя. В зависимости от переднего угла установки резца и формы и размера ядра сжатия изменяется и характер скола горной породы. Как правило, в мягких породах даже при малом усилии резца порода выходит из ядра сжатия на поверхность, поэтому ядро формируется слабоуплотненное и больших размеров. В твердых породах образовавшееся ядро по размерам меньше и имеет форму полусферы.

С одной стороны на ядро сжатия действует усилие резца $(R)$, а с другой - реакция еще не разрушенной горной породы $R_{\text {в }}$ (рис. 2). Как указано в работе [10], усилие $R_{\text {в }}$ определяется прочностными свойствами горной породы и зависит от скорости резанияскалывания породы резцом. Значение данного усилия можно определить по формуле (2):

$$
R_{\mathrm{B}}=\frac{\sigma_{\mathrm{c \kappa}} K \pi h^{\frac{3}{2}} \sqrt{d}}{2 \sin \gamma_{\text {ск }}},
$$

где $K$ - коэффициент, учитывающий повышение усилия сопротивления горной породы резанию-скалыванию при повышении линейной скорости резца.

Поэтому ядро сжатия представляет собой мелкораздробленную породу. Ю.И. Протасов описал такое состояние породы как «псевдожидкость». Такое определение подходит не только для твердых пород. Механизм разрушения мягких пород и пород средней твердости тоже подразумевает наличие на передней грани резца $P D C$ горной породы в уплотненном состоянии [6-12].

Так как обязательным условием процесса разрушения породы является образование ядра сжатия - 1 и трещины отрыва - 3, порода под режущей гранью резца разделяется на зоны, каждая из которых отличается своим состоянием и физико-механическими свойствами. Порода выше трещины отрыва считается разрушенной и отделяется от горного массива, превращаясь в шлам. Поэтому под резцом у забоя скважины, в месте, где промывочная жидкость встречается с разрушенной горной породой, образуется плотная зона обильного насыщения промывочной жидкости шламом - 4 (рис. 2). Именно в этой зоне происходит проникновение части промывочной жидкости в забой, что способствует дополнительному разупрочнению горной породы.

Таким образом, на резец оказывает влияние некая «жидкая среда», состоящая из смеси следующих компонентов:

- собственно промывочной жидкости - 5, плотность которой $\left(\rho_{\text {пж}}\right)$ зависит от принятого для бурения ее вида и в рассматриваемой зоне будет больше, чем на устье скважины;
- промывочной жидкости, обильно насыщенной шламом, плотность которой $\left(\rho_{\text {пж+ш }}\right)$ будет зависеть от вида промывочной жидкости и от свойств разрушаемой в данный момент породы;

- горной породы, плотность которой $\left(\rho_{\text {пор }}\right)$ зависит от ее физико-механических свойств и состояния.

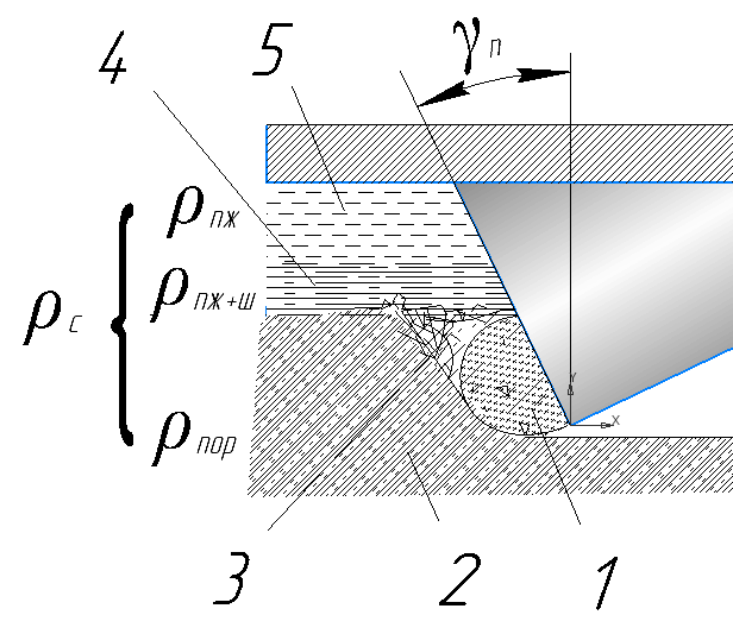

Pис. 2. Схема зоны контакта резия РDC с горной породой: 1 - ядро сжатия горной породы; 2 - неразрушенная горная порода; 3 - трещина отрыва; 4 - зона обильного насыщения промывочной жидкости шиламом; 5 - промывочная жидкость

Fig. 2. Diagram of the contact zone of the PDC cutter with the rock: 1 - rock compression core; 2 - undisturbed rock; 3 - separation crack; 4 - zone of abundant saturation of the flushing fluid with sludge; 5 -flushing fluid

На практике породоразрушающий инструмент типа $P D C$ применяется при бурении в горных породах, обладающих различной твердостью: мягких, средней твердости, а также в разрезах, где происходит переслаивание средних по твердости с пропластками твердых. Плотность таких пород колеблется в преде-

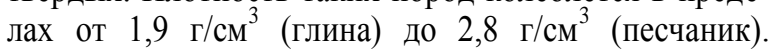
Удельная плотность разрушаемой горной породы может составить 20-25 кН/M ${ }^{3}$. Бурение таким инструментом возможно в условиях с промывкой растворами как на водной основе, плотность которых состав-

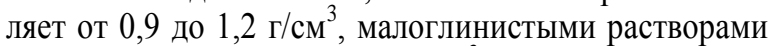
с плотностью $\rho=1,05 \ldots 1,06$ г $/ \mathrm{cm}^{3}$, так и на нефтяной основе $\left(\rho=0,89 \ldots 1 \Gamma / \mathrm{cm}^{3}\right)$. Следовательно, весовая плотность бурового раствора, насыщенного шламом, может быть в пределах от 11 до $14 \mathrm{\kappa H} / \mathrm{m}^{3}$.

Если рассмотреть область забоя строго под резцом, то можно выделить находящиеся под ним следующие зоны: зону неразрушенной горной породы; зону уплотненной и разрушенной горной породы; зону промывочной жидкости, насыщенной шламом, и зону прибывающей промывочной жидкости. Все эти составляющие оказывают влияние на свойства среды, находящейся в призабойной зоне скважины и оказывающей выталкивающее действие на резец. Учитывая возможные комбинации этих составляющих, весовая плотность рассматриваемой среды $\rho_{c}$ может находиться в широких пределах и составлять от 11 до 18 кН/ $/ \mathrm{m}^{3}$. 


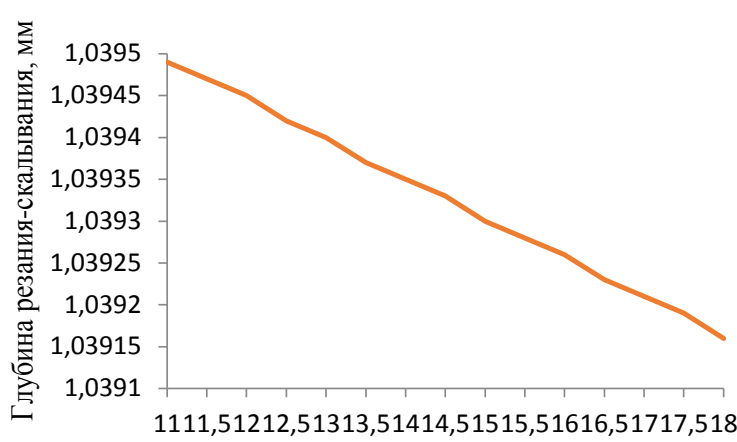

Весовая плотность среды, кН/м³

$a / a$

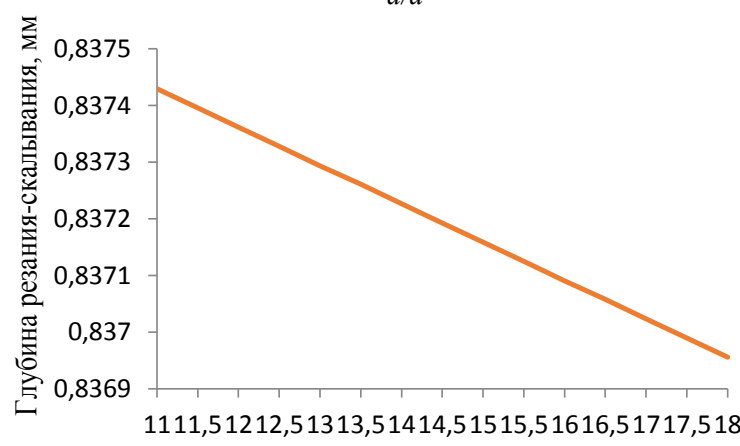

Весовая плотность среды, кН/м ${ }^{3}$ $\sigma / b$

Pис. 3. Зависимость глубины резания-скальвания породы резиом РDС от весовой плотности среды: а) диаметр резиа $15 \mathrm{Mм}$; б) диаметр резиа $20 \mathrm{Mм}$ $\left(\gamma_{n}=-20 ; v_{p}=4 \mathrm{M} / \mathrm{c}\right)$

Fig. 3. Dependence of the rock cutting-chipping depth with the PDC cutter on the medium weight density: a) cutter diameter $15 \mathrm{~mm}$; b) cutter diameter $20 \mathrm{~mm}$ $\left(\gamma_{n}=-20 ; v_{p}=4 \mathrm{~m} / \mathrm{s}\right)$

С целью определения степени влияния сопротивления представленной среды на эффективность разрушения горной породы резцом $P D C$ на основании формулы (1) с учетом возможных условий ее образования проведен расчет глубины резания-скалывания породы резцом при различных значениях плотности среды и аналитический анализ полученных результатов. В качестве исходных данных для расчета приняты усредненные для производственных условий значения параметров.

Результаты расчетов показали, что при прочих равных условиях в случае роста значения весовой плотности «жидкой среды» на 40 \% глубина резания породы резцом может уменьшиться на $0,2 \ldots 0,4 \%$ в зависимости от размера резца (рис. 3), что при общем количестве резцов в 20 шт. снизит значение углубления за оборот на $8 \%$.

При этом особое влияние на степень изменения показателя углубления оказывает величина линейной скорости перемещения резца. Результаты расчетов, учитывающих одинаковые условия бурения, показали, что при скорости $v_{\mathrm{p}}=2 \mathrm{M} / \mathrm{c}$ повышение весовой плотности на $40 \%$ приведет к снижению глубины резания резцом на $0,11 \%$, а при $v_{\mathrm{p}}=4 \mathrm{~m} / \mathrm{c}-$ на $0,3 \%$ (рис. 4).
Согласно графику, приведенному на рис. 4, при одновременном увеличении скорости резания и весовой плотности среды глубина резания начинает падать, при этом интенсивность падения ее значения и зависимость от плотности среды при большей $v_{\mathrm{p}}$ значительно выше. Полученный результат подтверждает положение ранее проведенных научных исследований [9], которое, дополнив результатами данной работы, можно заключить следующим образом: «по мере роста линейной скорости перемещения резца растет сопротивление среды, образовавшейся на забое скважины».

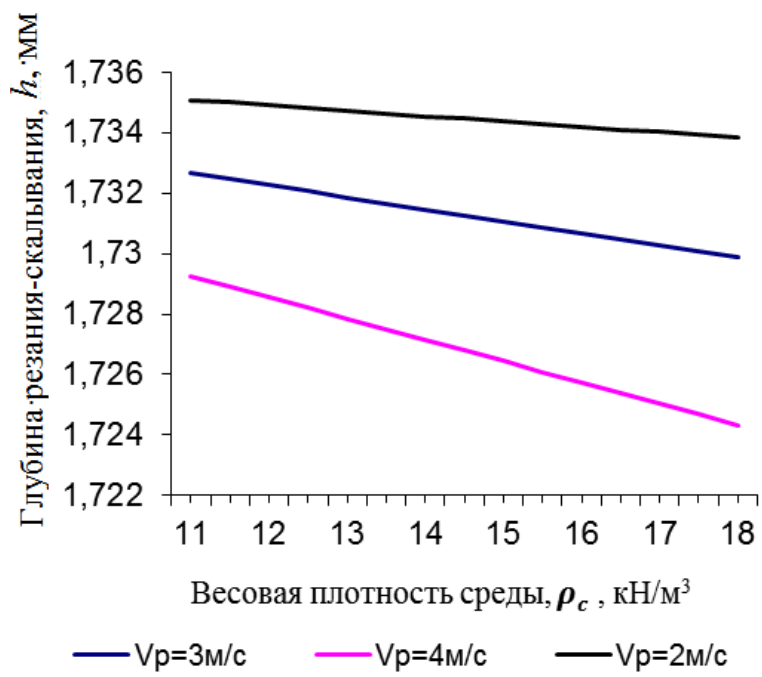

Рис. 4. Зависимость глубины резания-скалывания породы от весовой плотности жидкой среды $(d=15$ MM; $\gamma_{n}=-15$ )

Fig. 4. Rock cutting-chipping depth dependence on liquid medium weight density ( $\left.d=15 \mathrm{~mm} ; \gamma_{n}=-15\right)$

Как показали исследования гидродинамических процессов, протекающих на забое в процессе бурения породоразрушающим инструментом типа $P D C$ [11], перед резцом $P D C$ возникает область повышенного давления, созданная жидкой средой. Данную область можно разделить на три зоны, одна из которых, зона максимального давления III, располагается ближе к контакту резца с породой (рис. 5, a). Как показывают результаты компьютерного моделирования [11], чем больше передний угол установки резца, тем более распространенный по рабочей поверхности резца характер и повышенные значение при аналогичных параметрах бурения имеет зона максимального давления (рис. 5).

При этом аналитические расчеты по формуле (1) показывают, что чем больше значение переднего угла установки резца, тем большее влияние оказывает плотность среды на глубину его резания (рис. 6). Причем степень влияния может отличаться более чем в 1,5 раза.

Полученный результат указывает на взаимное влияние повышенного давления, вызванного изменением значения переднего угла установки резца, и плотности жидкой среды. 

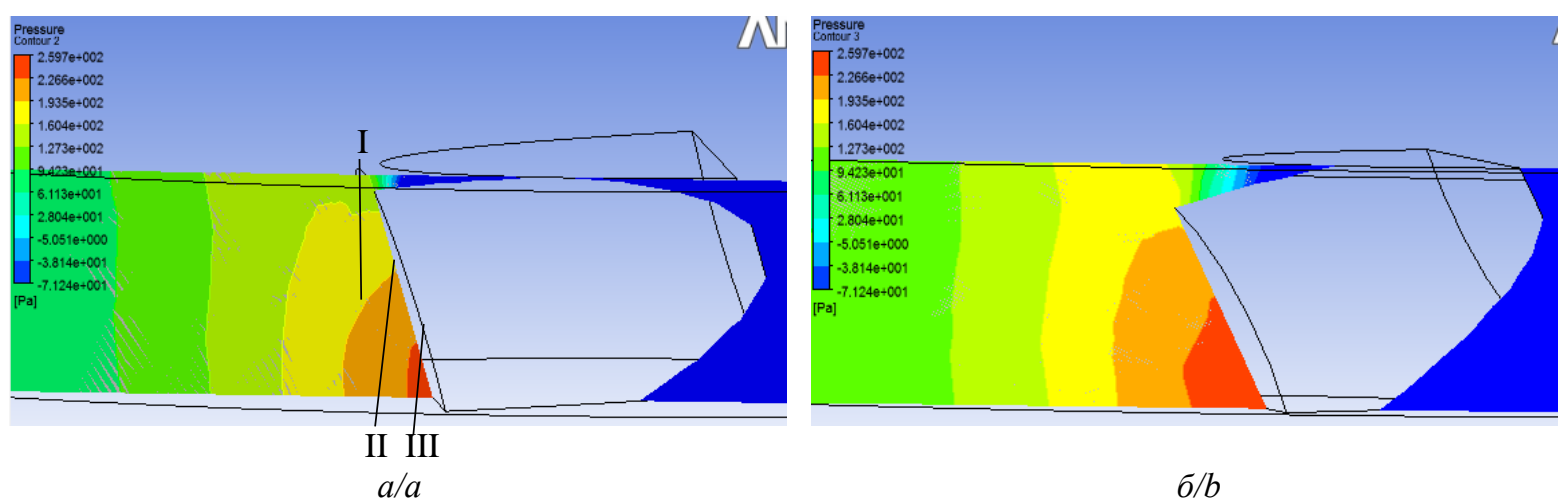

Рис. 5. Давление в пределах резиа PDC: a) $\gamma_{n}=-15^{\circ}$; б) $\gamma_{n}=-25^{\circ}$

Fig. 5. Pressure within the PDC cutter: a) $\gamma_{n}=-15^{\circ}$; b) $\gamma_{n}=-25^{\circ}$

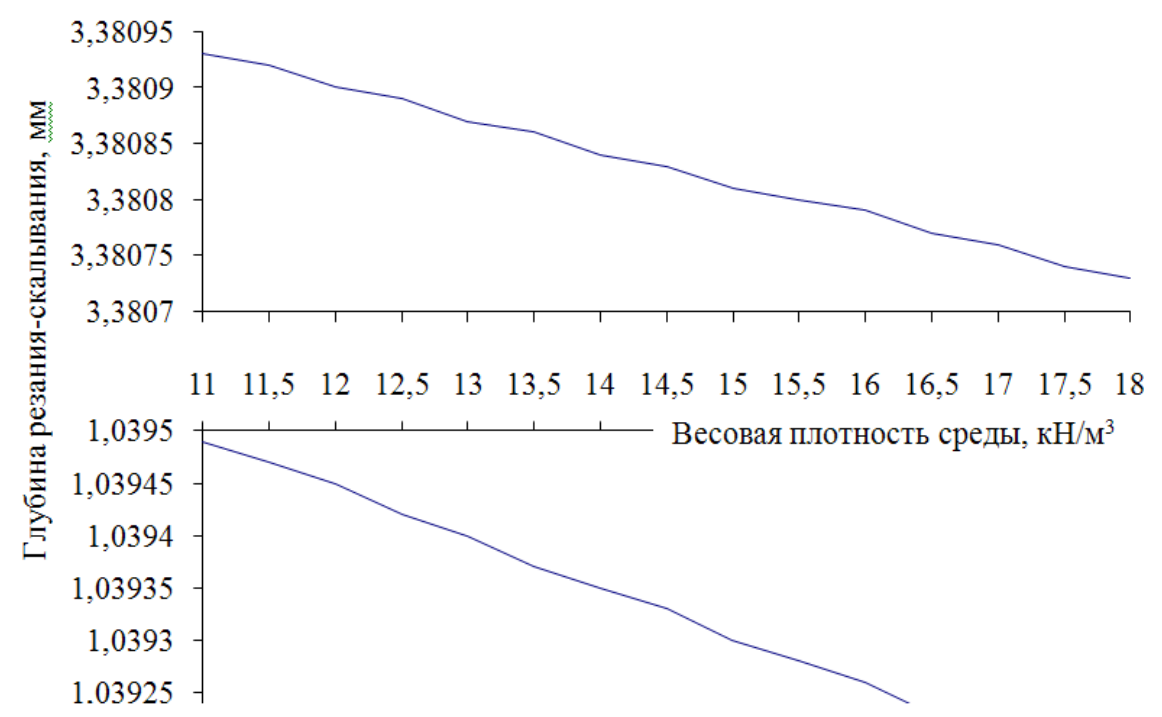

Pис. 6. Зависимость глубины резания-скалывания породы от весовой плотности жидкой среды: 1 - передний угол установки резиа $-10^{\circ} ; 2$ - передний угол установки резиа $-20^{\circ}$

Fig. 6. Rck cutting-chipping depth dependence on the liquid medium weight density: 1 - rake angle $-10^{\circ} ; 2$ - rake angle $20^{\circ}$

\section{Заключения, предложения}

Таким образом, среда, образовавшаяся в призабойной зоне скважины, оказывает сопротивление внедрению резца $P D C$ в горную породу, чем значительно снижает глубину резания-скалывания породы и как следствие понижает эффективность работы бурового инструмента.

Повышенное значение сопротивления среды объясняется ее давлением на режущую грань резца, а также плотностью ее состава.

Снижение сопротивления среды внедрению резца возможно путем борьбы с повышением ее плотности (например, за счет улучшения очистки забоя от шлама или применения в качестве промывочного агента ПАВ, воды), а также изменения конструктивных параметров армирования породоразрушающего инструмента резцами $P D C$. В качестве конструктивного метода можно выделить меры изменения величины переднего угла установки резца. Значения переднего угла рекомендуется принимать в пределах от -10 до $-20^{\circ}$.

Полученные результаты можно использовать также в направлении интенсификации разрушения гор- ной породы. Как известно, насыщение породы жидкостью способствует ее разупрочнению. Зона повышенного давления жидкой среды находится у контакта резца с породой, к этой же плоскости, согласно схеме на рис. 2, со стороны массива примыкает наиболее разрушенная часть породы - 3. Учитывая полученные данные, появляется возможность, путем управления струи подаваемой жидкости, повысить эффект насыщения этой зоны горной породы жидкостью. Одним из способов такого воздействия может выступить резец $P D C$ с вогнутой поверхностью его режущей грани. Форма резца с углублением в центре его режущей поверхности будет способствовать движению промывочного агента, поступающего из промывочных каналов долота, строго в центр режущей грани резца, что будет способствовать улучшению очистки от шлама самой нагруженной его части и направлению струи под давлением в массив горной породы. К тому же при бурении резцами вогнутой формы результирующая усилия резания-скалывания породы разделится на несколько векторов $R$, которые будут направлены на встречу друг другу (рис. 7). 
Противодействуя, усилия $R$ предотвратят поперечные колебания резца и таким образом будут способствовать гашению вибрации инструмента.

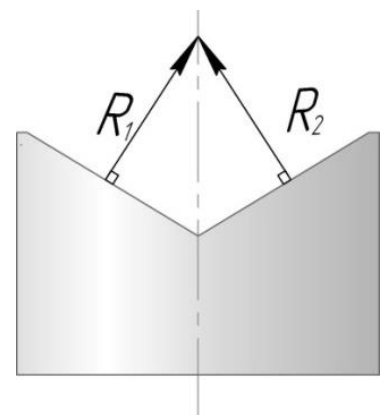

Рис. 7. Схема распределения усилия резиа с вогнутой режущей поверхностью при внедрении в породу (вид сверху)

Fig. 7. Diagram of distribution of the force of a cutter with a concave cutting surface when penetrating into the rock (top view)

\section{СПИСОК ЛИТЕРАТУРЫ}

1. Принципы конструирования и эксплуатации алмазного породоразрушающего инструмента / А.А. Буканов, Л.К. Горшков, А.И. Осецкий, Н.В. Соловьев // Разведка и охрана недр. 2013. - № 7. - С. 44-49.

2. Горшков Л.К., Осецкий А.И. Развитие принципов конструирования и эксплуатации нового алмазного породоразрушающего инструмента // Записки Горного института. - 2012. T. $197 .-$ C. $40-45$.

3. Brook B. Principles of diamond tool technology for sawing rock // International Journal of Rock Mechanics and Mining Sciences. 2002. - V. 39 (1). - P. 41-58.

4. Tonon F., Amadei B. Effect of elastic anisotropy on tunnel wall displacements behind a tunnel face // Rock Mechanics and Rock Engineering. - 2002. - V. 35 (3). - P. 141-160.

5. Нескоромных В.В. Разрушение горных пород при бурении скважин. - М.: ИНФРА-М; Красноярск: Сибирский федеральный университет, 2015. - 336 с.

6. Киселев А.Т., Крусир И.Н. Вращательно-ударное бурение геологоразведочных скважин. - М.: Недра, 1984. -102 с.

7. Detournay E., Richard T., Shepherd M. Drilling response of drag bits: theory and experiment // International Journal of Rock Mechanics and Mining Sciences. - 2008. - V. 45 (8). - P. 1347-1360.

8. Basarir H., Karpuz C. Preliminary estimation of rock mass strength using diamond bit drilling operational parameters // International Journal of Mining, Reclamation and Environment. 2016. - V. 30 (2). - P. 145-164.

9. Борисов К.И. Современные методы оценки сопротивления горных пород резанию-скалыванию при бурении долотами PDC: монография. - Томск: ТПУ, 2013. - $166 \mathrm{c.}$

10. Определение глубины разрушения горной породы резцами PDC с учетом сопротивления среды / В.В. Нескоромных, М.С. Попова, А.В. Чихоткин, А.Е. Головченко, Е.Е. Шубенина // Строительство нефтяных и газовых скважин на суше и на моpe. -2020 . - Т. 5 (329). - С. 23-27.

11. Нескоромных В.В., Попова М.С. Гидродинамика процесса резания резцами РDC // Строительство нефтяных и газовых скважин на суше и на море. - 2020. - Т. 7 (331). - С. 13-15.

12. Research on rock-breaking mechanism of cross-cutting PDC bit / Y. Yang, C. Zhang, M. Lin, L. Chen // J. Petrol. Sci. Eng. 2018. - V. 161. - P. 657-666.

13. Блинов Г.А., Васильев В.И., Глазов М.Г. Алмазосберегающая технология бурения. - Л.: Недра, 1989. - 184 с.

14. Нескоромных В.В. Оптимизация в геологоразведочном производстве. - Красноярск: Сибирский федеральный университет, 2013. $-246 \mathrm{c}$.

15. Нескоромных В.В., Пушмин П.С. Методика оптимальных параметров режима и условий бурения скважин // Известия Си-

\section{Выводы}

1. На основе схемы механизма разрушения и аналитической формулы определения глубины резанияскалывания породы резцом $P D C$ проведен анализ влияния образовывающейся на забое в процессе бурения среды на результативность работы породоразрушающего инструмента типа $P D C$.

2. Среда, образовавшаяся на забое, состоит из промывочной жидкости, шлама и разрушенной горной породы. Ее свойства зависят от вида используемой промывочной жидкости, характера воздействия резца на породу и параметры разрушаемой горной породы.

3. На эффективность разрушения горной породы резцом $P D C$ оказывает влияние плотность образованной на забое среды.

4. Минимизировать влияние «среды» можно путем понижения ее плотности, изменения геометрических параметров установки резца или улучшения очистки забоя.

бирского отделения Секции наук о Земле РАЕН - 2011. № 1 (38). - С. 151-157.

16. Инновационные подходы к конструированию высокоэффективного породоразрушающего инструмента / А.Я. Третьяк, В.В. Попов, А.Н. Гроссу, К.А. Борисов // Горный информационно-аналитический бюллетень. - 2017. - № 8. - С. 225-230.

17. Su O., Ali Akcin. Numerical simulation of rock cutting using the discrete element method // International Journal of Rock Mechanics and Mining Sciences. - 2011. - V. 48 (3). - P. 434-442.

18. Hasan A.R., Kabir C.S. Wellbore heat-transfer modeling and applications // Journal of Petroleum Science and Engineering. 2012. - V. 96-97. - P. 109-119.

19. A novel method for measuring and analyzing the interaction between drill bit and rock / X. Wang, Z. Wang, D. Wang, L. Chai // Measurement. - 2018. - V. 121. - P. 344-354.

20. Kanyanta V., Ozbayraktar S., Maweja K. Effect of manufacturing parameters on polycrystalline diamond compact cutting tool stressstate // International Journal of Refractory Metals and Hard Materials. - 2014. - V. 45. - P. 147-152

21. Che D., Zhu W.-L., Ehmann K.F. Chipping and crushing mechanisms in orthogonal rock cutting // International Journal of Mechanical Sciences. - 2016. - № 119. - P. 224-236.

22. Евсеев В.Д., Мавлютов М.Р. Пути повышения эффективности разрушения горных пород // Материалы региональной конференции геологов Сибири, Дальнего Востока и Северо-Востока России. - 2000. - T. I. - С. 463-465.

23. Борисов К.И. Актуальные научные и прикладные вопросы развития нового научного метода оценки свойств и эффективности динамического разрушения горных пород инструментами режуще-скалывающего типа PDC // Вестник Ассоциации буровых подрядчиков. - 2010. - № 3-С. 8-14.

24. Нескоромных В.В., Попова М.С., Харитонов А.Ю. Влияние сил сопротивления на глубину резания-скалывания горной породы алмазным резцом // Известия Томского политехнического университета. Инжиниринг георесурсов. - 2020. - Т. 331. - № 10. - С. $40-48$.

25. Closed-form solution of stress state and stability analysis of wellbore in anisotropic permeable rocks / D.-P. Do, N.-H. Tran, H.-L. Dang, D. Hoxha // International Journal of Rock Mechanics and Mining Sciences. - 2019. - V. 113. - P. 11-23.

26. Ai Z., Han Y., Kuang Y., Wang Y., Zhang M. Optimization model for polycrystalline diamond compact bits based on reverse design // Advances in Mechanical Engineering. - 2018. - V. 10 (6). - P. 476-479.

27. Numerical investigations on the effect of ultra-high cutting speed on the cutting heat and rock-breaking performance of a single cutter / M. Gao, K. Zhang, Q. Zhou, H. Zhou, B. Liu, G. Zheng // Journal of Petroleum Science and Engineering. - July 2020. - V. 190. - № 107120.

Поступила 18.08.2021 2. 


\section{Информация об авторах}

Нескоромных В.B., доктор технических наук, профессор, заведующий кафедрой технологии и техники разведки Института горного дела, геологии и геотехнологий Сибирского федерального университета.

Попова M.C., кандидат технических наук, доцент кафедры технологии и техники разведки Института горного дела, геологии и геотехнологий Сибирского федерального университета.

Баочанг Л., профессор геоинженерии кафедры разведки и бурения. 
UDC 622.24.051.64

\title{
INFLUENCE OF THE BOTTOM BOREHOLE ZONE ENVIRONMENT ON THE EFFICIENCY OF ROCK BREAKING BY THE PDC CUTTER
}

\author{
Vyacheslav V. Neskoromnykh', \\ sovair@bk.ru
}

\author{
Marina S. Popova1, \\ alleniram83@mail.ru \\ Liu Baochang², \\ liubc@jlu.edu.cn

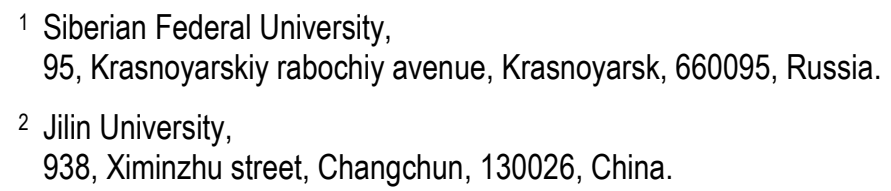

Relevance. The PDC cutter reinforced rock cutting tool has proven itself as a rock cutting tool with good performance. As it is known from drilling practice, a tool of this type, with the correct technology of its application, is capable of providing high rates of penetration of wells and resource. Therefore, one of the priority directions in the development of technical means of drilling is to improve the design of the PDC-type tool. New generation drilling tool design methods should be based on an integrated approach to the study of its work at the bottom. One of the main factors affecting the effectiveness of the implementation of rock cutting tools is the bottomhole resistance. A large number of works have been devoted to the study of changes in the physical and mechanical properties of a geological section with depth. And the liquid medium formed at the bottom of the well as a result of drilling is poorly studied. Often, when conducting scientific research, the presence of cuttings in the bottomhole zone is neglected. However, taking into account the peculiarities of the shape and size of the cutting part of the PDC cutters, the displacement of cuttings and continuously moving drilling fluid can have certain density and create a buoyant force acting on the plane of the cutter and, as a result, affect the efficiency of the drilling tool as a whole. Therefore, an urgent issue is to study the properties of the media, consisting of a flushing agent, cuttings and destroyed rock and determine the degree of its influence on the results of the PDC-type drilling tool.

The purpose of the research is to determine the degree of influence of the resistance forces of the medium formed in the bottomhole zone of the well on well drilling.

Methods: analytical method, computer simulation method.

Results. The medium formed in the near-wellbore zone when drilling a well and consisting of rock, cuttings and flushing fluid, which are in different states, has a pushing effect on the cutter, which reduces the size of the deepening. The index of the resistance degree of the described medium is determined by the cutting speed, the angle of the cutter and the density of its components. The results obtained can be used in the design of a PDC-type drilling tool. Changing the angle of installation or the shape of the cutter can help not only to reduce the resistance forces, but also to improve the destructive ability of the tool due to the decompaction of the rock by intensifying liquid penetration into the rock.

\section{Key words:}

Cutting-chipping speed, deepening, drilling, PDC cutter, medium resistance, cutting-chipping depth, compression core, rock failure.

\section{REFERENCES}

1. Bukanov A.A., Gorshkov L.K., Osetskiy A.I., Soloviev N.V. Printsipy konstruirovaniya i ekspluatatsii almaznogo porodorazrushayushchego instrumenta [Principles for the design and operation of diamond rock cutting tools]. Razvedka i ohrana nedr, 2013, no. 7, pp. 44-49.

2. Gorshkov L.K., Osetskiy A.I. Development of principles for the design and operation of a new diamond rock cutting tool. Journal of Mining Institute, 2012, vol. 197, pp. 40-45. In Rus.

3. Brook B. Principles of diamond tool technology for sawing rock International Journal of Rock Mechanics and Mining Sciences, 2002, vol. 39 (1), pp. 41-58.

4. Tonon F., Amadei B. Effect of elastic anisotropy on tunnel wall displacements behind a tunnel face. Rock Mechanics and Rock Engineering, 2002, vol. 35, no. 3, pp. 141-160.

5. Neskoromnykh V.V. Razrushenie gornykh porod pri burenii skvazhin [Rock destruction when drilling]. Moscow, INFRA-M; Krasnoyarsk, SFU, 2015. $336 \mathrm{p}$.

6. Kiselev A.T., Krusir I.N. Vrashchatelno-udarnoe burenie geologorazvedochnykh skvazhin [Rotary-shock drilling of exploration wells]. Moscow, Nedra Publ., 1984. 102 p.

7. Detournay E., Richard T., Shepherd M. Drilling response of drag bits: theory and experiment. International Journal of Rock Mechanics and Mining Sciences, 2008, vol. 45 (8), pp. 1347-1360.
8. Basarir H., Karpuz C. Preliminary estimation of rock mass strength using diamond bit drilling operational parameters. International Journal of Mining, Reclamation and Environment, 2016, vol. 30 (2), pp. 145-164.

9. Borisov K.I. Sovremennye metody otsenki soprotivleniya gornykh porod rezaniyu-skalyvaniyu pri burenii dolotami PDC. Monografiya [Modern methods for assessing the resistance of rocks to cutting-chipping when drilling with PDC bits. Monograph]. Tomsk, TPU Publ. house, 2013. 166 p.

10. Neskoromnih V.V., Popova M.S., Chikhotkin A.V., Golovchenko A.E., Shubenina E.E. Opredelenie glubiny razrusheniya gornoy porody reztsami PDC $\mathrm{s}$ uchetom soprotivleniya sredy [Determination of the depth of destruction of rocks with PDC cutters, taking into account the resistance of the medium]. Construction of oil and gas wells on land and sea, 2020, vol. 5 (329), pp. 23-27.

11. Neskoromnih V.V., Popova M.S. Development of a drilling process control technique based on a comprehensive analysis of the criteria. Journal of Mining Institute, 2019, vol. 240, pp. 701710. In Rus. DOI: 10.31897/pmi.2019.6.701.

12. Yang Y., Zhang C., Lin M., Chen L. Research on rock-breaking mechanism of cross-cutting PDC bit. J. Petrol. Sci. Eng., 2018, vol. 161, pp. 657-666. 
13. Blinov G.A., Vasiliev V.I., Glazov M.G. Almazosberegayushchaya tekhnologiya bureniya [Diamond-saving drilling technology]. Leningrad, Nedra Publ., 1989. 184 p.

14. Neskoromnykh V.V. Optimizatsiya $v$ geologorazvedochnom proizvodstve [Optimization in geological survey production]. Moscow, INFRA-M Publ.; Krasnoyarsk, SFU Publ., 2013. 246 p.

15. Neskoromnyh V.V., Pushmin P.S. Metodika optimalnykh parametrov rezhima i uslovy bureniya skvazhin [Methodology of optimal parameters of the mode and conditions of well drilling]. Izvestiya Sibirskogo otdeleniya Sektsii nauk o Zemle RAEN, 2011, no. 1 (38), pp. 151-157.

16. Tretyak A.Ya., Popov V.V., Grossu A.N., Borisov K.A. Innovative approaches to the design of highly efficient rock cutting tools. Gorny informatsionno-analiticheskii byulleten, 2017, no. 8, pp. 225-230. In Rus.

17. Su O., Ali Akcin. Numerucal simulation of rock cutting using the discrete element method. International Journal of Rock Mechanics and Mining Sciences, 2011, vol. 48 (3), pp. 434-442.

18. Hasan A.R., Kabir C.S. Wellbore heat-transfer modeling and applications. Journal of Petroleum Science and Engineering, 2012, vol. 96-97, pp. 109-119.

19. Wang X., Wang Z., Wang D., Chai L. A novel method for measuring and analyzing the interaction between drill bit and rock. Measurement, 2018, vol. 121, pp. 344-354.

20. Kanyanta V., Ozbayraktar S., Maweja K. Effect of manufacturing parameters on polycrystalline diamond compact cutting tool stressstate. International Journal of Refractory Metals and Hard Materials, 2014, no. 45, pp. 147-152.

21. Che D., Zhu W.-L., Ehmann K.F. Chipping and crushing mechanisms in orthogonal rock cutting. International Journal of Mechanical Sciences, 2016, no. 119, pp. 224-236.
22. Evseev V.D., Mavlyutov M.R. Puti povysheniya effektivnosti razrusheniya gornykh porod [Ways to increase the efficiency of rock destruction]. Materialy regionalnoy konferentsii geologov Sibiri, Dalnego Vostoka i Severo-Vostoka Rossii, 2000, vol. I, pp. 463.

23. Borisov K.I. Aktualnye nauchnye i prikladnye voprosy razvitiya novogo nauchnogo metoda otsenki svoystv $\mathrm{i}$ effektivnosti dinamicheskogo razrusheniya gornykh porod instrumentami rezhushche-skalyvayushchego tipa PDC [Actual scientific and applied issues of the development of a new scientific method for assessing the properties and efficiency of dynamic destruction of rocks with tools of cutting-shearing type PDC]. Vestnik Associatsii burovykh podryadchikov, 2010, no. 3, pp. 8-14.

24. Neskoromnykh V.V., Popova M.S., Kharitonov A.Yu. Influence of resistance forces on the depth of rock cutting-chipping by a diamond cutter. Bulletin of the Tomsk Polytechnic University. Geo Assets Engineering, 2020, vol. 331, no. 10, pp. 40-48. In Rus.

25. Do D.-P., Tran N.-H., Dang H.-L., Hoxha D. Closed-form solution of stress state and stability analysis of wellbore in anisotropic permeable rocks. International Journal of Rock Mechanics and Mining Sciences, 2019, vol. 113, pp. 11-23.

26. Ai Z., Han Y., Kuang Y., Wang Y., Zhang M. Optimization model for polycrystalline diamond compact bits based on reverse design. Advances in Mechanical Engineering, 2018, 10 (6), pp. 476-479.

27. Gao M., Zhang K., Zhou Q., Zhou H., Liu B., Zheng G. Numerical investigations on the effect of ultra-high cutting speed on the cutting heat and rock-breaking performance of a single cutter. Journal of Petroleum Science and Engineering, July 2020, vol. 190, article no. 107120.

Received: 18 August 2021.

\section{Information about the authors}

Vyacheslav V. Neskoromnykh, Dr. Sc., professor, head of the department of technology and equipment of investigation, Siberian Federal University.

Marina S. Popova, Cand. Sc., associate professor, Siberian Federal University.

Liu Baochang, professor, Jilin University. 